Part of Journal of Research of the National Bureau of Standards, Volume 34, July 1945

\title{
ACETYL DERIVATIVES OF CERTAIN HEPTOSES, OF GULOSE, AND OF LACTULOSE
}

\author{
By Harriet L. Frush and Horace S. Isbell
}

\begin{abstract}
In the course of an investigation of the stereomeric factors that influence the course of replacement reactions, several new acetyl derivatives of the sugars were prepared. Inasmuch as the sugar acetates are of importance for analytical purposes, for the synthesis of other substances, and for the elucidation of structure, the new compounds are reported, even though the study of the reaction mechanisms has been temporarily discontinued. The preparation and properties are given for several new acetates of the heptoses, and for acetates of D-gulose, D-glucoheptulose, and lactulose.

The following new compounds are reported: Hexaacetyl-D-glycero- $\beta-D-i d o-$ aldoheptose, melting point $136^{\circ} \mathrm{C},[a]_{D}^{20},-9.1^{\circ}$; pentaacetyl-D-glycero- $\alpha-\mathrm{D}-i d o-$ aldoheptosyl bromide, melting point $114^{\circ} \mathrm{C},[a]_{D}^{20},+143^{\circ}$; methyl pentaacetyl-Dglycero- $\beta$-D-ido-aldoheptoside, melting point $136^{\circ}$ to $137^{\circ} \mathrm{C},[a]_{D}^{20},-31.3^{\circ}$; hexaacetyl-D-glycero- $a$-D-talo-aldoheptose, melting point $137^{\circ} \mathrm{C},[a]_{D}^{20},+88.8^{\circ}$; hexaacetyl-D-glycero-L-galacto-aldoheptoses (crystalline mixture); pentaacetyl-D-gulose, melting point $113^{\circ} \mathrm{C},[a]_{D}^{20},+86.2^{\circ}$ (needle form), and melting point $105^{\circ}$ to $106^{\circ} \mathrm{C},[a]_{D}^{20},+86.2^{\circ}$ (prismatic form); pentaacetyl-D-glucoheptulose, melting point $114^{\circ}$ to $115^{\circ} \mathrm{C},[a]_{D}^{20},+45.9^{\circ}$; octaacetyllactulose, melting point $138^{\circ} \mathrm{C}$, $[a]_{D}^{20},-6.6^{\circ}$.
\end{abstract}

\section{CONTENTS}

I. Introduction

II. Derivatives of D-glycero-D-ido-aldoheptose (D- $\beta$-glucoheptose) $\ldots$

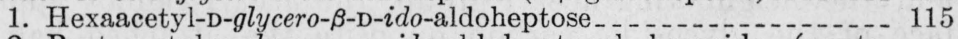

2. Pentaacetyl-D-glycero- $a$-D-ido-aldoheptosyl bromide (pentaacetyl- $a$-D- $\beta$-glucoheptosyl bromide) $\ldots \ldots \ldots$

3. Methyl pentaacetyl-D-glycero- $\beta$-D-ido-aldoheptoside

III. Hexaacetyl-D-glycero- $\alpha$-D-talo-aldoheptose (hexaacetyl- $\alpha-D-\beta$-mannoheptose)

IV. Hexaacetyl-D-glycero-L-galacto-aldoheptoses (hexaacetyl-D- $\beta$-guloheptoses) _ .

V. Pentaacetyl-D-gulose

VI. Pentaacetyl-D-glucoheptulose

VII. Octaacetyllactulose

VIII. References _. .

\section{INTRODUCTION}

In the cyclic sugar derivatives the carbon atoms have known configurations and are united in a ring, so that rotation about the individual carbon-carbon bonds cannot take place. Consequently, the spheres of influence of the various groups attached to the ring are definitely fixed in relation to one another. For this reason the cyclic sugar derivatives are particularly suitable for studying the 
effect of the relative position in space of the groups within the molecule upon the course and mechanism of replacement reactions.

Several years ago an investigation was begun in this laboratory of the influence of acetyl groups attached to the pyranose ring on the course of replacement reactions $[1,2]{ }^{1} \quad$ At that time it was planned to prepare a large number of acetylglycosyl halides and to study their behavior in the Koenigs-Knorr reaction in order to ascertain the part which acetyl groups of neighboring carbons play in replacement reactions. A sufficient number of compounds was studied to warrant the formulation of the hypothesis that when an acetyl group is capable of approaching the face of the glycosidic carbon opposite the attached halogen, cleavage of the halogen occurs with Walden inversion and the formation of an orthoester. ${ }^{2}$ Further study of the Koenigs-Knorr reaction has been in progress for some time, but because of the national emergency this work has been discontinued for the present. It seems desirable, however, to report the preparation and properties of certain new compounds that have been obtained, even though the work is not complete.

According to the premise on which the investigation was based, an acetylglycosyl halide having a trans configuration for the halogen of the glycosidic carbon and the acetyl group of the adjacent carbon can yield, on application of the Koenigs-Knorr reaction, the alpha and beta glycosides, and a methyl orthoacetate. Reaction 1 shows the formation of a glycoside with inversion of the glycosidic carbon; reaction 2 shows the formation of a methyl orthoacetate; whereas reaction 3 shows the formation of a methyl glycoside having the same configuration as the parent acetylglycosyl halide. Reaction 3 involves the formation of an intermediate of the orthoester type; as it includes two inversions, the configuration of the glycosidic carbon is retained. Although reaction 3 is illustrated in the diagram with a transient intermediate of the orthoester type, other transient intermediates are possible and might be formed from nucleophilic (electron-sharing) groups in the environment.

In an acetylglycosyl halide having a cis configuration for the halogen and the neighboring acetyl group, the acetyl cannot approach the face of the glycosidic carbon opposite the attached halogen, and consequently it cannot directly undergo reactions of the orthoester type. The halogen can be replaced readily, with inversion, by nucleophilic groups from the environment. The formation, in some cases, of small quantities of the glycoside having the same configuration as the parent acetylglycosyl halide may arise from an even number of successive displacements with inversion involving one or more unstable intermediates. In addition to the mechanisms outlined above, reactions resulting in racemization may take place by the formation of free carbonium ions. This course seems, however, to be of little importance under the conditions used in the present investigation.

\footnotetext{
1 Figures in brackets indicate the literature references at the end of this paper.

3 An excellent review of the subject of carbohydrate orthoesters is given by H. W. Post in The chemistry of aliphatic orthoesters, p. 106-114 (Reinhold Publishing Corporation, New York, N. Y., 1943).
} 
In the aldoses, carbon 1 is bound in a ring, and consequently only those acetylglycosyl halides having the acetyl group of carbon 2 and the halide of carbon 1 in the trans position form orthoesters. In the ketoses, the halogen of the acetylglycosyl halide is located on carbon 2 , and may be replaced by the acetyl groups of either carbon 1 or carbon 3. Presumably, reaction of an acetyl group of carbon 3 is

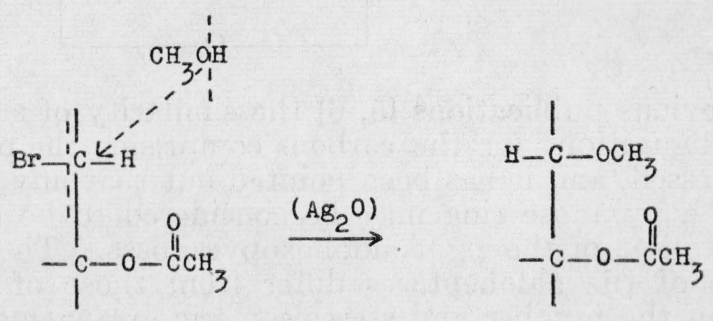<smiles>CC(C)OC(=O)OC(C)(C)Br</smiles><smiles>COC1(C)OCC(C)O1</smiles>

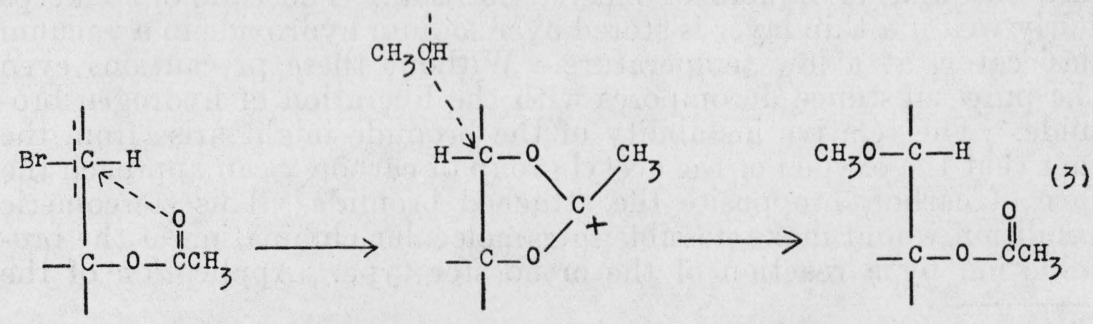

possible only when the group is trans to the halogen of carbon 2 . As carbon 1 of the ketoses is not bound in the ring, and rotation about the carbon-carbon bond is possible, an acetyl group attached to carbon 1 can always approach carbon 2 from the direction favorable for orthoester formation. Consequently, all the ketoses should be capable of forming 1,2-orthoesters. Several orthoesters of ketoses have been reported in the literature [3], but none has been proved to have the 2,3-structure, and further investigation seems desirable. 


\section{DERIVATIVES OF D-GLYCERO-D-IDO-ALDOHEPTOSE} $\left(D-\beta\right.$-GLUCOHEPTOSE) ${ }^{3}$ [4]<smiles>OCC(O)C(O)C(O)CO</smiles>

In previous publications [5, 6] the similarity of substances having like configurations for the carbons comprising the pyranose ring has been stressed, and it has been pointed out that any sugar capable of forming a pyranose ring may be considered to be configurationally related to one of the eight aldohexopyranoses. The pyranose modifications of the aldoheptoses differ from those of the aldohexoses merely in the number and stereoisomeric arrangement of the atoms in the side chain attached to the pyranose ring.

Little is known concerning the properties of the compounds of idose, the only aldohexose which has not been obtained in the crystalline state. ${ }^{4}$ However, D-glycero-D-ido-aldoheptose (D- $\beta$-glucoheptose), one of the two aldoheptoses having the idose configuration, has been crystallized [8]. In this investigation a crystalline acetate, acetylglycosyl bromide, and methyl acetylglycoside of the sugar have been prepared. The acetyl- $\alpha$-glycosyl bromide is of particular interest to the present study because the acetyl group of carbon 2 is trans to the halogen of carbon 1 and can approach the face of carbon 1 from the direction presumably favorable for the production of orthoesters.

The experimental study was complicated by the fact that the acetylglycosyl bromide is relatively unstable. When it was dissolved in chloroform, a mutarotation reaction took place with gradual discoloration of the solution. The compound could not be reclaimed from the chloroform solution, which turned black overnight, as did also the mother liquors of recrystallization. The compound keeps fairly well if a thin layer is stored over sodium hydroxide in a vacuum desiccator, at a low temperature. Without these precautions even the pure substance decomposes with the liberation of hydrogen bromide. The relative instability of the bromide might arise from the fact that the oxygen of the acetyl group of carbon 2 can approach the face of carbon 1 opposite the attached bromide. This stereomeric condition would make possible intramolecular elimination of the bromide ion by a reaction of the orthoester type. Application of the

3 The systematic names used in this paper are derived by a procedure which is applicable to substances containing more than four contiguous asymmetric carbon atoms. The horizontal formula is written in the Fischer conventional manner with the lowest-numbered asymmetric carbon at the right. Configurational units are set off in order, beginning with the lowest-numbered asymmetric carbon, and the name is read from left to right in manner analogous to setting off and reading a large number. The derivation of the systematic name for $\mathrm{D}$ - $\beta$-glucoheptose is illustrated below:

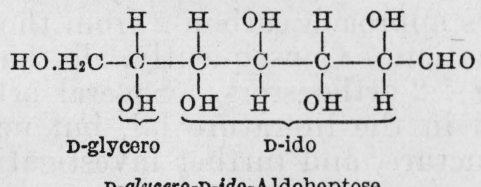

4 Gulose has been crystallized only in the form of a calcium chloride compound [7 
Koenigs-Knorr reaction [9] to the acetylglycosyl bromide gave a product that was obviously a mixture. From this mixture a crystalline glycoside, tentatively classified as the beta pyranose modification, was obtained in 43-percent yield; the residual products of the reaction have not yet been identified, but will be the subject of future investigation.

A comparison by means of Hudson's isorotation principle [10] of the molecular rotations of the three new derivatives of D-glycero-D-idoaldoheptose with molecular rotations of previously known pyranose derivatives of the epimeric sugar, D-glycero-D-gulo-aldoheptose is of interest.

\begin{tabular}{|c|c|c|}
\hline $\begin{array}{l}\text { Hexaacetyl-D-glycero- } \beta \text {-D-gulo-aldoheptose } \\
\text { Hexaacetyl-D-glycero-D-ido-aldoheptose...- }\end{array}$ & $\begin{array}{l}{[\alpha]_{D}} \\
+4.8^{\circ} \\
-9.1^{\circ}\end{array}$ & $\begin{aligned} & {[M]_{D} } \\
&+ 2,200 \\
&- 4,200 \\
&\end{aligned}$ \\
\hline Difference & & $+6,400$ \\
\hline $\begin{array}{l}\text { Pentaacetyl-D-glycero- } \alpha \text {-D-gulo-aldoheptosyl } \\
\text { bromide. } \\
\text { Pentaacetyl-D-glycero-D-ido-aldoheptosyl bro- } \\
\text { mide_. }\end{array}$ & $\begin{array}{l}+156^{\circ} \\
+143^{\circ}\end{array}$ & $\begin{array}{l}+75,400 \\
+69,100\end{array}$ \\
\hline Difference........ & & $+6,300$ \\
\hline $\begin{array}{l}\text { Methyl pentaacetyl-D-glycero- } \beta \text {-D-gulo-aldo- } \\
\text { heptoside. } \\
\text { Methyl pentaacetyl-D-glycero-D-ido-aldohep- } \\
\text { toside }\end{array}$ & $\begin{array}{l}-16^{\circ} \\
-31.3^{\circ}\end{array}$ & $\begin{array}{r}-6,950 \\
-13,600\end{array}$ \\
\hline
\end{tabular}

Refer[11]

Pentaacetyl-D-glycero- $\alpha$-D-gulo-aldoheptosyl

Pentaacetyl-D-glycero-D-ido-aldoheptosyl bro-

mide $+69,100$

Methyl pentaacetyl-D-glycero- $\beta$-D-gulo-aldoheptoside.

Difference

$$
\begin{array}{lr}
-16^{\circ} & -6,950 \\
-31.3^{\circ} & -13,600 \\
\hline & +6,650
\end{array}
$$

The fact that pyranoses are ordinarily obtained by the methods used for preparing the new compounds, and the approximate agreement of the epimeric differences, seem to support the classification of the new compounds as pyranose derivatives. Apparently the acetate and acetylglycoside are beta compounds, and the bromide is an alpha compound. Additional work is necessary for the rigorous classification of these substances, but they are tentatively designated:

Hexaacetyl-D-glycero- $\beta$-D- $i d o$-aldoheptose.

Pentaacetyl-D-glycero- $\alpha$-D-ido-aldoheptosyl bromide.

Methyl pentaacetyl-D-glycero- $\beta$-D- $i d o$-aldoheptoside.

\section{HEXAACETYL-D-GLYCERO- $\beta$-D-IDO-ALDOHEPTOSE}

Twenty-five grams of finely powdered D-glycero-D-ido-aldoheptose (D- $\beta$-glucoheptose) [8] was added to a mixture of $300 \mathrm{ml}$ of acetic anhydride and $250 \mathrm{ml}$ of pyridine previously cooled to $0^{\circ} \mathrm{C}$ and contained in a flask equipped with a sealed mechanical stirrer. The mixture was stirred at $0^{\circ} \mathrm{C}$ until solution was complete (about 2 days) and then poured with stirring into 6 liters of ice water. After half an hour, the product was extracted with chloroform, and the chloroform extract was washed several times with an aqueous solution of cupric nitrate and finally with water. The chloroform solution was dried, filtered, and finally concentrated in vacuum to a sirup from which the acetate crystallized. After the addition of ether the crystals were separated by filtration. By concentrating the mother liquor, a second crop of crystals was obtained, making a total yield of $36.7 \mathrm{~g}$ of crude acetate. Three successive crystallizations from warm ethyl alcohol 
yielded pure hexaacetyl-D-glycero-D-ido-aldoheptose, $\mathrm{mp}, 136^{\circ} \mathrm{C}$. $[\alpha]_{D}^{20}=-9.1^{\circ}$ (chloroform, $\left.c=4\right) .^{5}$ The melting point and optical rotation were unchanged by recrystallization. A microphotograph of the crystals is given in figure $1, A$.

Analysis: Calculated for $\mathrm{C}_{19} \mathrm{H}_{26} \mathrm{O}_{13}: \mathrm{C}, 49.35 ; \mathrm{H}, 5.67 ; \mathrm{CH}_{3} \mathrm{CO}$, 55.85. Found: C, $49.2 ; \mathrm{H}, 5.6 ; \mathrm{CH}_{3} \mathrm{CO}, 55.8$.

\section{PENTAACETYL-D-GLYCERO- $\alpha$-D-IDO-ALDOHEPTOSYL BROMIDE (PENTAACETYL- $\alpha$-D- $\beta$-GLUCOHEPTOSYL BROMIDE)}

One gram of finely powdered hexaacetyl-D-glycero- $\beta$-D-ido-aldoheptose was brominated at $0^{\circ} \mathrm{C}$ by treating it with $5 \mathrm{ml}$ of a saturated solution of hydrogen bromide in acetic acid, to which $0.5 \mathrm{ml}$ of acetic anhydride had been added. The sugar dissolved in about 10 minutes. The solution was kept at $0^{\circ} \mathrm{C}$ for 3 hours, after which time it was poured into ice water, and extracted quickly with benzene. The benzene extract was washed six times with ice water, dried with Drierite, and after filtration, concentrated in vacuum to a thin sirup, which crystalsized upon the addition of ether. The preparation was repeated on leveral 5-g quantities of hexaacetyl-D-glycero- $\beta$-D-ido-aldoheptose, which yielded about $4 \mathrm{~g}$ each of the crude bromide. The compound was recrystallized several times by dissolving it in ether, and adding petroleum ether to saturation. When pure, it melts at $114^{\circ} \mathrm{C}$.

Analysis: Calculated for $\mathrm{C}_{17} \mathrm{H}_{23} \mathrm{O}_{11} \mathrm{Br}$ : C, $42.25 ; \mathrm{H}, 4.80 ; \mathrm{Br}, 16.54$. Found: $\mathrm{C}, 42.9 ; \mathrm{H}, 4.8 ; \mathrm{Br}, 16.3$.

A $0.0490-\mathrm{g}$ sample of the substance dissolved in purified chloroform to give a volume of $1.511 \mathrm{ml}$ showed an optical rotation in a 1-dm tube of $+13.39^{\circ} \mathrm{S} 10$ minutes after dissolution. The optical rotation decreased in 5 hours to $+10.43^{\circ} \mathrm{S}$; after 18 hours the solution was too dark to read. The reading 10 minutes after dissolution corresponds to $[\alpha]_{D}^{20}=+143^{\circ}$ (chloroform, $c=3.2$ ). A microphotograph of the the crystals is given in figure $1, B$.

\section{METHYL PENTAACETYL-D-GLYCERO- $\beta$-D-IDO-ALDOHEPTOSIDE}

Four grams of D-glycero- $\alpha$-D-ido-aldoheptosyl bromide was added to a cold reaction mixture consisting of $200 \mathrm{ml}$ of anhydrous methyl alcohol, $16 \mathrm{~g}$ of freshly prepared silver carbonate, and $8 \mathrm{~g}$ of powdered Drierite. The mixture was stirred continuously at $0^{\circ} \mathrm{C}$ for about 1 day, and then filtered. The filtrate was concentrated in vacuum to a sirup, from which approximately $3 \mathrm{~g}$ of crystals was separated. Examination of the material under the microscope revealed two types of crystals. After two recrystallizations from ethyl alcohol, $1.55 \mathrm{~g}$ of homogeneous material, $\mathrm{mp} 136^{\circ}$ to $137^{\circ} \mathrm{C}$, was obtained. $[\alpha]_{D}^{20}=-31.3^{\circ}$ (chloroform, $c=2)$. The constants were not changed by further recrystallization. A microphotograph of the crystals is given in figure $1, C$.

Analysis: Calculated for $\mathrm{C}_{18} \mathrm{H}_{26} \mathrm{O}_{12}: \mathrm{C}, 49.77 ; \mathrm{H}, 6.03 ; \mathrm{CH}_{3} \mathrm{CO}$, 49.56; $\mathrm{CH}_{3} \mathrm{O}, 7.14$. Found: C, 50.0; H, 6.1; $\mathrm{CH}_{3} \mathrm{CO}, 49.7 ; \mathrm{CH}_{3} \mathrm{O}, 7.1$.

In order to ascertain whether or not the substance is an orthoester, it was treated with a 0.5 -percent solution of hydrogen chloride in dry chloroform under conditions which have been shown to cause the rapid conversion of orthoesters to acetylglycosyl chlorides [13]. See also page 420 of [2]. No rapid change in optical rotation was ob-

The constants for this substance were previously reported on page 724 of reference [10b]. 
served, and after 10 minutes the hydrogen chloride was removed by the addition of dry silver carbonate and filtration. After evaporation of the filtrate, crystalline methyl pentaacetyl-D-glycero- $\beta$-D-idoaldoheptoside was reclaimed unchanged. As the substance in chloroform solution is not sensitive to hydrogen chloride, it does not have an orthoester structure.

\section{HEXAACETYL-D-GLYCERO- $\alpha$-D-TALO-ALDOHEPTOSE (HEXAACETYL- $\alpha$-D- $\beta$-MANNOHEPTOSE)}

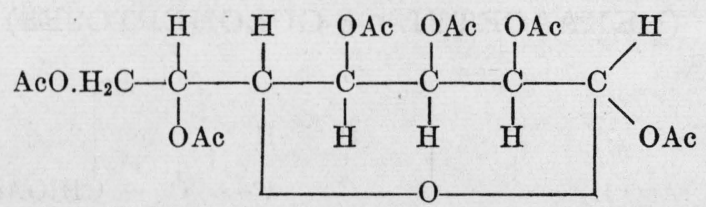

D-glycero-D-talo-Aldoheptose $[14,15]$ is one of the two sugars obtained by application of the cyanhydrin synthesis to mannose. The free sugar has the $\alpha$-D-talose configuration for the atoms comprising the pyranose ring, and its properties show a parallelism to those of $\alpha$-D-talose. As tetraacetyl- $\alpha$-D-talosyl bromide is readily obtained in the crystalline state and gives rise by the Koenigs-Knorr reaction to a methyl orthoacetate [16], it was of interest to attempt the successive preparation of the acetate, acetylglycosyl bromide, and the methyl orthoacetate of D-glycero-D-talo-aldoheptose. The acetate has been prepared, and its properties are reported here, but the preparation of the acetylglycosyl bromide and the study of its behavior in the Koenigs-Knorr reaction must await a more opportune time.

Inasmuch as the new acetate was prepared from D-glycero- $\alpha$-D-taloaldoheptopyranose, and contains six acetyl groups, it is presumably hexaacetyl-D-glycero- $\alpha$-D-talo-aldoheptopyranose. This classification is supported by the agreement of its optical rotation with that calculated from the rotations of compounds affording a comparison of "epimeric differences" [17]. D-glycero-D-talo-Aldoheptose (D- $\beta$ - mannoheptose) has the D-talose structure, and D-glycero-D-galacto-aldoheptose (D- $\alpha$-mannoheptose) has the D-galactose structure. The molecular rotation of pentaacetyl- $\alpha$-D-talopyranose $(+27,400)[16]$ is 14,250 units less than that of pentaacetyl- $\alpha$-D-galactopyranose $(+41,650)$ [18]. Assuming that this epimeric difference applies in the heptose series, the molecular rotation of hexaacetyl-D-glycero- $\alpha$-Dtalo-aldoheptopyranose should be 14,250 units less than that of hexaacetyl-D-glycero- $\alpha$-D-galacto-aldoheptopyranose $(+55,850)$ [19], or $+41,600$. This value corresponds to a specific rotation of $+90.0^{\circ}$ in comparison with the observed value of $+88.8^{\circ}$.

Acetylation of $\mathrm{D}$-glycero- $\alpha$-D-talo-aldoheptose.-A finely powdered sample of D-glycero- $\alpha$-D-talo-aldoheptose monohydrate $(2.35 \mathrm{~g})$ was acetylated at $0^{\circ} \mathrm{C}$ with a mixture of $13 \mathrm{ml}$ of acetic anhydride and 20 $\mathrm{ml}$ of pyridine. The mixture was stirred at $0^{\circ} \mathrm{C}$ for 18 hours, and then poured with stirring into $400 \mathrm{ml}$ of ice water. In the course of half an hour the product crystallized in small needles or slender prisms, which, when separated, weighed $3.6 \mathrm{~g}$ and melted at $128^{\circ}$ to $132^{\circ} \mathrm{C}$. The crude product was recrystallized from $15 \mathrm{ml}$ of hot ethyl alcohol. The first crop of crystals weighed $2.7 \mathrm{~g}$ and melted at 
$135^{\circ}$ to $137^{\circ} \mathrm{C}$. Recrystallization from $10 \mathrm{ml}$ of hot ethyl alcohol gave $2.5 \mathrm{~g}$, melting at $137^{\circ} \mathrm{C}$ (fig. $1, A$ ). $[\alpha]_{D}^{20}=+88.8^{\circ}$ (chloroform, $c=4$ ). The melting point and optical rotation were unchanged by further recrystallization of the compound.

Analysis: Calculated for $\mathrm{C}_{19} \mathrm{H}_{26} \mathrm{O}_{13}: \mathrm{C}, 49.35 ; \mathrm{H}, 5.67 ; \mathrm{CH}_{3} \mathrm{CO}$, 55.85. Found: $\mathrm{C}, 49.6 ; \mathrm{H}, 5.7 ; \mathrm{CH}_{3} \mathrm{CO}, 55.6$.

\section{HEXAACETYL-D-GLYCERO-L-GALACTO- ALDOHEPTOSES}

\section{(HEXAACETYL-D- $\beta$-GULOHEPTOSES)}<smiles>COC(C)C(COC(C)=O)C(C)CC(C)OC(C)=O</smiles>

Acetylation of D-glycero-L-galacto-aldoheptose.-A $1.35 \mathrm{gm}$ sample of finely powdered D-glycero- $\alpha$-L-galacto-aldoheptose $\left(\alpha\right.$-D- $\beta$-guloheptose) ${ }^{6}$ was acetylated at $0^{\circ} \mathrm{C}$ with $9 \mathrm{ml}$ of acetic anhdride and $14 \mathrm{ml}$ of pyridine. The sugar went into solution slowly; when solution was complete the mixture was poured with stirring into 10 volumes of ice water. In the course of about half an hour, crystals separated from the water mixture; they were collected on a filter and the filtrate was extracted with benzene. The benzene solution was evaporated to a sirup, which was brought to crystallization by the addition of alcohol. The crystals obtained both from water and from the benzene extract melted over a wide range and appeared to be mixtures. By numerous recrystallizations of the combined products from ethyl, isopropyl, and isoamyl alcohols, and finally from ligroin, two fractions were obtained, the larger of which melted at $117^{\circ}$ to $118^{\circ} \mathrm{C}$ and had a specific rotation of $-65^{\circ}$. A smaller fraction melted at $150^{\circ}$ to $155^{\circ} \mathrm{C}$ and had a specific rotation of approximately $-15^{\circ}$. Microscopic examination of the fractions showed that they were not homogeneous and consisted of mixtures of large flat plates and slender needles. However, it has been impossible with the amount of material at hand to effect a complete separation and purification of these acetates. The analysis of the larger fraction (mp, $117^{\circ}$ to $118^{\circ} \mathrm{C},[\alpha]_{D}^{20},-65^{\circ}$ ) agrees with that of a hexaacetylheptose. Hence it is assumed that the material consists of two crystalline isomeric hexaacetates of D-glycero-L-galactoaldoheptose. The formation of two crystalline acetates shows that the conversion of the parent sugar to other modifications takes place during the acetylation, even under the mild conditions employed here.

Analysis of the fraction melting at $117^{\circ}$ to $118^{\circ} \mathrm{C}$ : Calculated for $\mathrm{C}_{19} \mathrm{H}_{26} \mathrm{O}_{13}:$ C, $49.35 ; \mathrm{H}, 5.67 ; \mathrm{CH}_{3} \mathrm{CO}$, 55.85. Found: $\mathrm{C}, 49.3 ; \mathrm{H}$, 5.7; $\mathrm{CH}_{3} \mathrm{CO}, 55.3$.

\footnotetext{
- LaForge [20], who was the first to prepare this sugar in the crystalline state, called it $\alpha$-d-guloheptose. For reasons pointed out in [21] the name was subsequently changed by Isbell to $d-\beta$-guloheptose. The systematic name for the alpha modification of the sugar is D-glycero- $\alpha$-L-galacto-aldoheptose.
} 


\section{PENTAACETYL-D-GULOSE ${ }^{7}$}

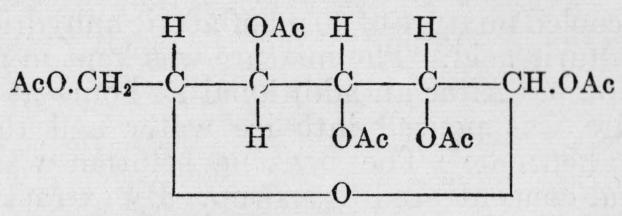

The aldohexose, gulose, has been crystallized only in the form of the calcium chloride compounds, $\alpha$-D-gulose. $\mathrm{CaCl}_{2} \cdot \mathrm{H}_{2} \mathrm{O}$ and (Dgulose $)_{2} \cdot \mathrm{CaCl}_{2}$ [7]. Numerous attempts to obtain a crystalline acetate by acetylation of $\alpha$-D-gulose. $\mathrm{CaCl}_{2} \cdot \mathrm{H}_{2} \mathrm{O}$ either with acetic anhydride and pyridine or with acetic anhydride and sodium acetate gave sirups that failed to crystallize. Acetylation of the calcium chloride compound with acetic anhydride and sulfuric acid at $0^{\circ} \mathrm{C}$ gave a small quantity of a crystalline gulose pentaacetate. The material was obtained in somewhat higher yield by the zinc chloride method.

A comparison, by means of Hudson's principle of isorotation, of the molecular rotation of pentaacetyl-D-gulose with the molecular rotations of some configurationally related substances may be used for the tentative assignment of structure to the compound. Thus $[M]_{D}$ of hexaacetyl-D-glycero- $\alpha$-D-gulo-aldoheptopyranose minus $[M]_{D}$ of methyl pentaacetyl-D-glycero- $\alpha$-D-gulo-aldoheptopyranoside should equal $[M]_{D}$ of pentaacetyl- $\alpha$-D-gulopyranose minus $[M]_{D}$ of methyl tetraacetyl- $\alpha$-Dgulopyranoside.

Unfortunately, two values are reported in the literature for the molecular rotation of methyl pentaacetyl-D-glycero- $\alpha$-D-gulo-aldoheptopyranoside. One of these was obtained for a product derived by application of the Koenigs-Knorr reaction to pentaacetyl-D-glycero$\beta$-D-gulo-aldoheptopyranosyl chloride [12] and the other for a produet derived by acetylation of crystalline methyl D-glycero- $\alpha$-D-guloaldoheptopyranoside [6]. It is of interest to calculate the optical rotation of pentaacetyl- $\alpha$-D-gulopyranose from the relation indicated above by the use of each of the two values for the molecular rotation of methyl pentaacetyl-D-glycero- $\alpha$-D-gulo-aldoheptopyranoside. The fol-

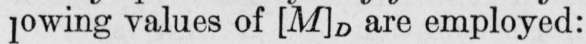

$$
\begin{aligned}
& \text { Hexaacetyl-D-glycero- } \alpha \text {-D-gulo-aldoheptopyranose } \ldots+. .+40,250 \text { [11] } \\
& \text { Methyl pentaacetyl-D-glycero- } \alpha \text {-D-gulo-aldoheptopy- }\{+39,550 \text { [12] } \\
& \text { ranoside........... } \\
& \text { Methyl tetraacetyl- } \alpha \text {-D-gulopyranoside....... }
\end{aligned}
$$

These values give a molecular rotation for pentaacetyl- $\alpha$-D-gulopyranose of $+35,950$ based on the value of Haworth, Hirst, and Stacey [12], and of $+28,850$ based on the value of Isbell and Frush [6]. They correspond to specific rotations of $+92.1^{\circ}$ and $+73.9^{\circ}$, respectively, in comparison with the observed specific rotation of $+86.2^{\circ}$. The observed rotation lies sufficiently close to the calculated rotations to warrant the tentative assignment of the alpha pyranose structure to the crystalline pentaacetate of gulose. The difference in the values may arise from the different ring conformations previously suggested by us as a possible explanation of other discrepancies in the optical rotations of substances in the gulose and D-glycero-D-gulo-aldoheptose series [6, p. 134].

\footnotetext{
${ }^{7}$ The structure shown here is to be considered as tentative.
} 
Acetylation of $\alpha$-D-gulose. $\mathrm{CaCl}_{2} \cdot \mathrm{H}_{2} \mathrm{O}$ with acetic anhydride and sulfuric acid.-Ten grams of finely powdered $\alpha$-D-gulose. $\mathrm{CaCl}_{2} \cdot \mathrm{H}_{2} \mathrm{O}$ was added to a previously cooled mixture of $60 \mathrm{ml}$ of acetic anhydride and $2.5 \mathrm{ml}$ of concentrated sulfuric acid. The mixture was kept in an ice bath and stirred for 24 hours. After an additional 24 hours at room temperature the mixture was poured into ice water and the product was extracted with benzene. The benzene solution was washed with water, dried, and concentrated to a sirup. By extraction of the sirup with hot water and evaporation of the water solution, a small quantity of crystalline gulose pentaacetate was obtained. ${ }^{8}$ This material was used for seeding the sirups obtained by the zinc chloride method.

Acetylation of $\alpha$-D-gulose. $\mathrm{CaCl}_{2} \cdot \mathrm{H}_{2} \mathrm{O}$ with acetic anhydride and zinc chloride. -Ten grams of finely powdered $\alpha$-D-gulose- $\mathrm{CaCl}_{2} \cdot \mathrm{H}_{2} \mathrm{O}$ was added to $50 \mathrm{ml}$ of ice-cold acetic anhydride containing $15 \mathrm{~g}$ of anhydrous zinc chloride and $20 \mathrm{~g}$ of dry sand. The mixture was placed in an ice bath and stirred until the gulose calcium chloride went into solution $(24 \mathrm{hr})$. The solution was then poured into ice water and the product was extracted with chloroform. The chloroform solution was washed with water and dried with sodium sulfate. On evaporation it gave a nearly colorless sirup which was diluted with $20 \mathrm{ml}$ of secondary amyl alcohol and seeded with crystalline gulose pentaacetate. The solution was placed in a refrigerator and was allowed to stand until crystallization was complete, after which the crystals were separated and washed with amyl alcohol. The yield by this procedure varied from 1 to $4 \mathrm{~g}$, and efforts to obtain higher yields and more consistent results were unsuccessful.

In order to obtain a larger quantity of the acetate, $150 \mathrm{~g}$-of $\alpha$-Dgulose. $\mathrm{CaCl}_{2} \cdot \mathrm{H}_{2} \mathrm{O}$ was treated with $700 \mathrm{ml}$ of acetic anhydride and $225 \mathrm{~g}$ of freshly fused zinc chloride. The mixture was mechanically stirred at $0^{\circ} \mathrm{C}$ for 5 days, and at room temperature for 5 days, and then poured with stirring into 8 liters of ice and water. The product was extracted with benzene and the benzene solution was washed with water, dried, and concentrated under reduced pressure to a sirup. The sirupy acetate was dissolved in 10 liters of boiling water; the solution was cooled, seeded with the crystalline acetate, and allowed to evaporate in air at room temperature. As evaporation proceeded, large crystals grew in the solution and a thin layer of sirupy acetate formed on the surface of the liquid. From time to time this layer was mechanically removed to avoid mixing with the crystals. Ultimately a total of $50 \mathrm{~g}$ of the crystalline gulose pentaacetate was separated from the liquid in the form of transparent hexagonal plates, some of which were nearly one-fourth inch in diameter.

When the compound was recrystallized from warm ethyl alcohol it formed slender needles $\left(\mathrm{mp}, 113^{\circ} \mathrm{C},[\alpha]_{D}^{20},+86.2^{\circ}\right)$. However, the needle crystals (fig. 2, A) were unstable and upon standing, or occasionally even during separation by filtration, changed to chunky truncated prisms $\left(\mathrm{mp}, 105^{\circ}\right.$ to $106^{\circ} \mathrm{C}$; fig. $\left.2, B\right)$. Because of the difficulty in obtaining a single crystalline modification, alcohol was abandoned as a solvent, and the acetate was recrystallized from water.

\footnotetext{
8 This compound was first crystallized in 1931, but was not closely investigated. The melting point of one form and the optical rotation were reported on page 736 of reference [10b].
} 

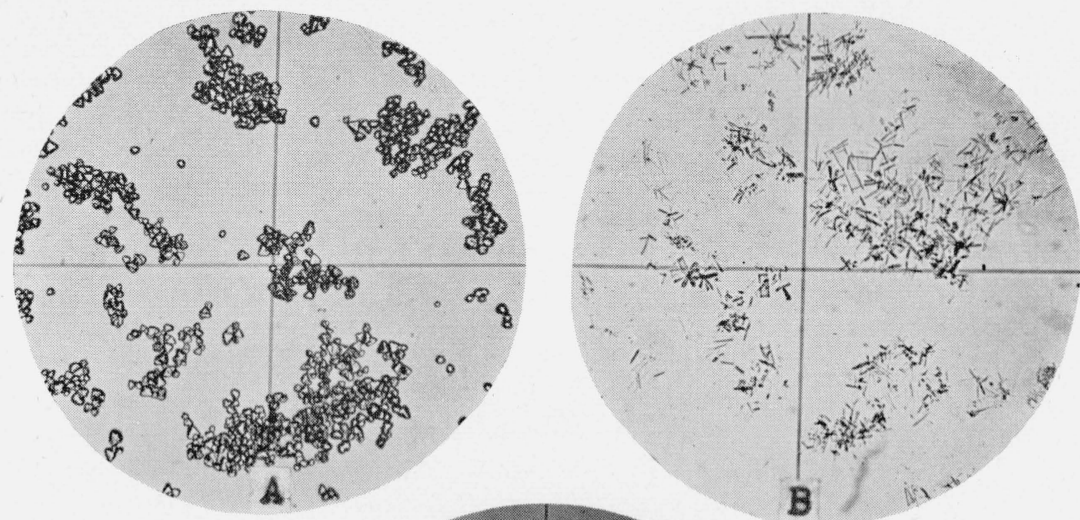

B

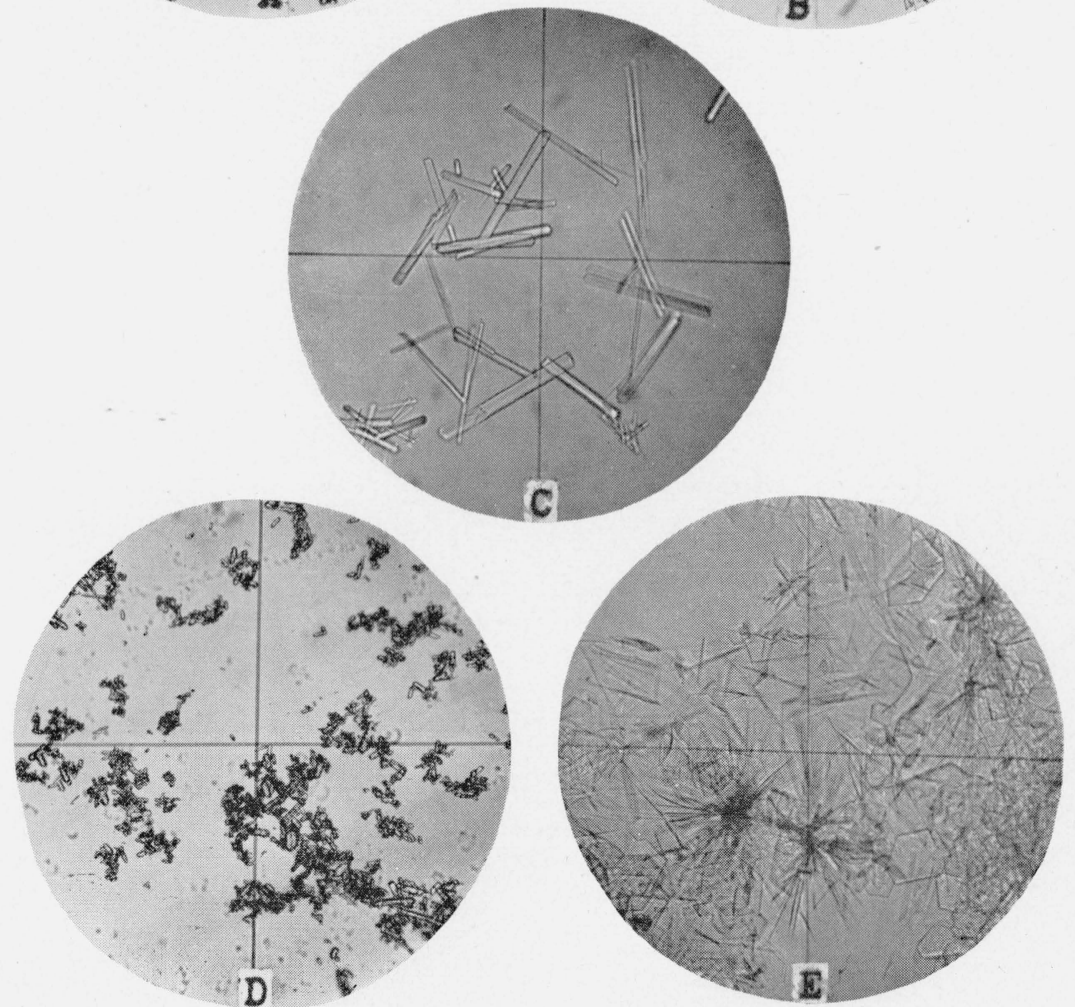

FiguRE 1.-Photomicrographs of new compounds.

$A$, Hexaacetyl-D-glycero- $\beta$-D-ido-aldoheptose (hexaacetyl- $\beta$-D- $\beta$-glucoheptose); $B$, pentaacetyl-D-glycer 0 - $\alpha$-Dido-aldoheptosyl bromide (pentaacetyl- $\alpha$-D- $\beta$-glucoheptosyl bromide); $C$, methyl pentaacetyl-D-glycero- $\beta$ D-ido-aldoheptoside (methyl pentaacetyl- $\beta$-D- $\beta$-glucoheptoside); $D$, hexaacetyl-D-glycer 0 - $\alpha$-D-talo-aldoheptose (hexaacetyl- $\alpha$-D- $\beta$-mannoheptose); $E$, exaacetyl-D-glycer 0 -L-galacto-aldoheptoses (hexaacetyl-D- $\beta$ guloheptoses). 

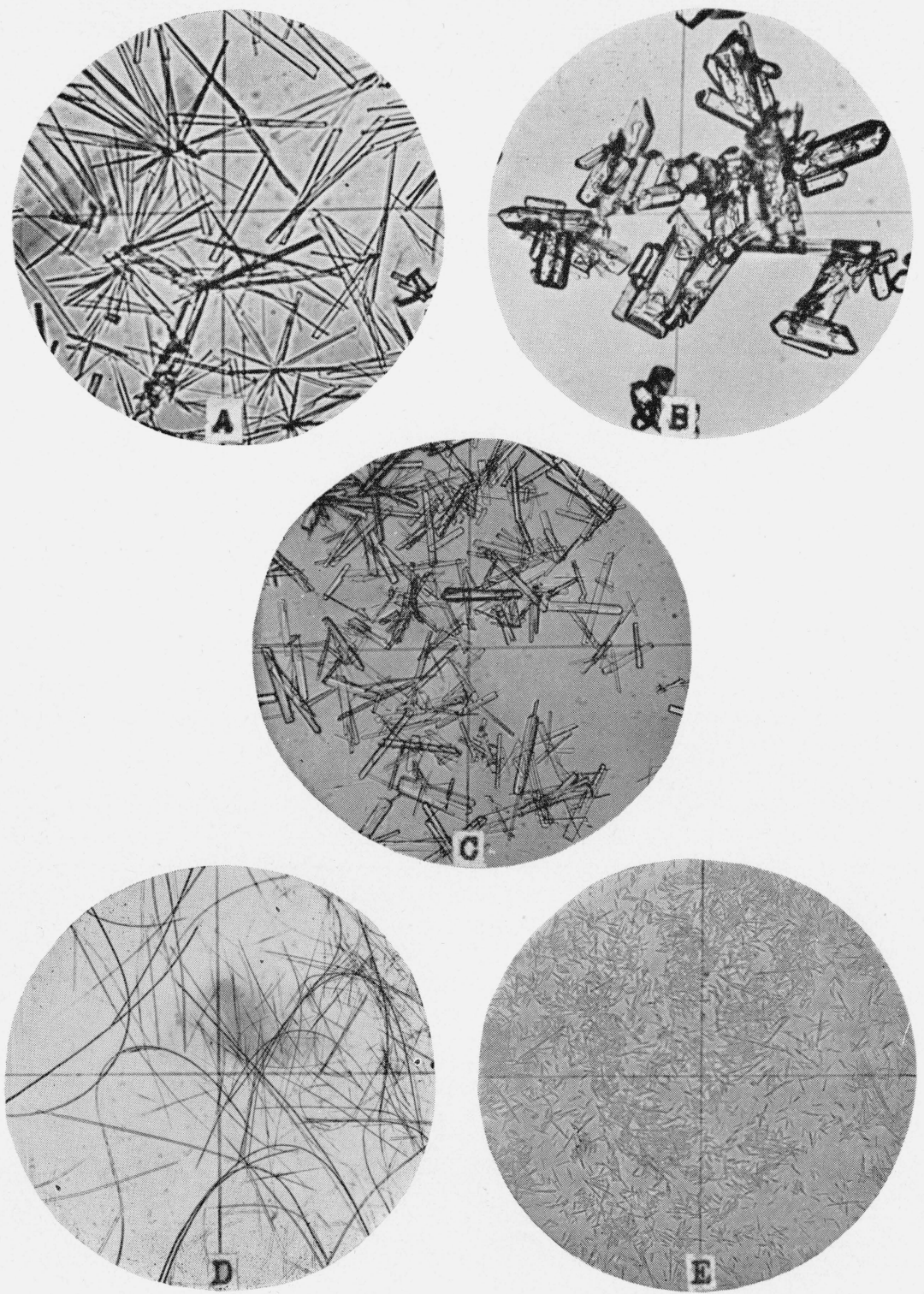

FigORE 2.-Photomicrographs of new compounds.

$A$, Pentaacetyl-D-gulose (needle form); $B$, pentaacetyl-D-gulose (prismatic form); $C$, pentaacetyl-D-glucoheptulose; $D$, octaacetyllactulose; $E$, octaacetyllactulose (crystallized in motion). 
The material was dissolved in hot water and the solution was concentrated in vacuum to approximately one-fourth of its volume. On cooling, it yielded exclusively the modification melting at $105^{\circ}$ to $106^{\circ} \mathrm{C}$. $[\alpha]_{D}^{20}=+86.2^{\circ}$ (chloroform, $c=2$ ).

Analysis: Calculated for $\mathrm{C}_{16} \mathrm{H}_{22} \mathrm{O}_{11}: \mathrm{C}, 49.23 ; \mathrm{H}, 5.68 ; \mathrm{CH}_{3} \mathrm{CO}, 55.14$. Found for the needle crystals $\left(\mathrm{mp}, 113^{\circ} \mathrm{C}\right)$ : C, 49.3; H, 5.6. Found for the prismatic crystals $\left(\mathrm{mp}, 105^{\circ}\right.$ to $\left.106^{\circ} \mathrm{C}\right): \mathrm{C}, 49.4 ; \mathrm{H}, 5.6$; $\mathrm{CH}_{3} \mathrm{CO}, 55.3$.

\section{PENTAACETYL-D-GLUCOHEPTULOSE ${ }^{9}$}

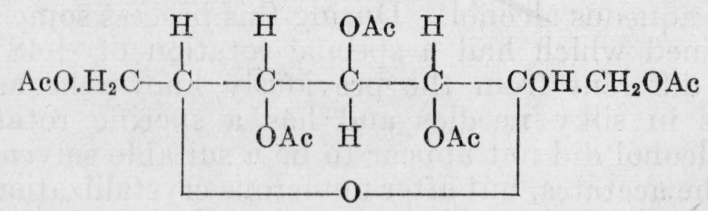

Prior to this investigation, Austin [23] had reported the preparation of hexaacetyl- $\alpha$-D-glucoheptulopyranose from the corresponding sugar by the sodium acetate method of acetylation. It was expected that the same acetate would be formed by application of the milder pyridine method. However, the crystalline product obtained by the latter method proved to be a mixture from which has been isolated, in addition to hexaacetyl- $\alpha$-D-glucoheptulose, a new pentaacetate and a crude fraction having an optical rotation lower than that of either. Acetylation of the new pentaacetate gave the previously known hexaacetyl- $\alpha$-D-glucoheptulopyranose. Hence the pentaacetylglucoheptulose has the pyranose ring.

The pyranose modifications of D-glucoheptulose and D-sorbose have the same configuration for the atoms comprising the ring, but differ in that the heptose has a $\mathrm{CH}_{2} \mathrm{OH}$ group attached to carbon 6 in place of a hydrogen atom. By acetylation of sorbose under approximately the same conditions used in acetylating D-glucoheptulose, a tetraacetate having a free glycosidic hydroxyl group is formed [24]. By analogy, one might expect the new compound of glucoheptulose to have a free hydroxyl group on carbon 2 and to be 1,3,4,5,7-pentaacetylD-glucoheptulose. Assuming that the glycosidic hydroxyl is free, an indication of the configuration of the glycosidic carbon may be gained through a comparison of molecular rotations. By application of Hudson's isorotation principle to the molecular rotations of methyl tetraacetyl- $\alpha$-D-sorbopyranoside $(+19,050)$ [24], 1,3,4,5-tetraacetyl$\alpha$-D-sorbose $(+7,400)[24],{ }^{10}$ and methyl pentaacetyl- $\alpha$-D-gluco-heptulopyranoside $(+34,100)$ [23], the molecular rotation of $1,3,4,5,7-$ pentaacetyl- $\alpha$-D-glucoheptulose is calculated to be $+22,450$. This value corresponds to a specific rotation of $+53.4^{\circ}$, in comparison with the observed value of $+45.9^{\circ}$. The agreement between the observed value and the calculated rotation is not sufficiently close to warrant a definite assignment of structure, although the compound is probably 1,3,4,5,7-pentaacetyl- $\alpha$-D-glucoheptulose. The assignment of the acetyl groups to definite positions, however, is left to future investigation, and the compound is designated merely as pentaacetyl-D-glucoheptulopyranose.

Structure is tentative.

10 The molecular rotations of the D-sorbose derivatives were derived from those of the corresponding derivatives of L-Sorbose. 


\section{Journal of Research of the National Bureau of Standards}

Acetylation of D-glucoheptulose.-Twenty grams of powdered Dglucoheptulose was added to $92 \mathrm{ml}$ of acetic anhydride and $140 \mathrm{ml}$ of pyridine, previously cooled to $0^{\circ} \mathrm{C}$. The mixture was stirred at $0^{\circ} \mathrm{C}$ for 3 days and at room temperature for 2 days. It was then poured, with stirring, into ice water and the produce was extracted with chloroform. The chloroform solution was concentrated in vacuum to a thick sirup, which was brought to crystallization by the addition of ether and petroleum ether. The crude acetate weighed about $30 \mathrm{~g}$ and the melting point was approximately $80^{\circ} \mathrm{C}$. The material, which proved to be a mixture, was recrystallized several times from 50 -percent aqueous alcohol. During this process some slender prisms were obtained which had a specific rotation of $+48^{\circ}$. They were obviously different from the previously known hexaacetate, which crystallizes in silky needles and has a specific rotation of $+87^{\circ}$. Aqueous alcohol did not appear to be a suitable solvent for the separation of the acetates, but after numerous crystallizations from carbon tetrachloride, and determinations of optical rotation, the material was separated into three fractions consisting of the previously known hexaacetyl- $\alpha$-D-glucoheptulose, a new pentaacetate, and a fraction having a specific rotation lower than that of either $\left(+10^{\circ}\right)$. The new pentaacetate crystallizes in slender prisms (fig. 2, $C$ ), which melt at $114^{\circ}$ to $115^{\circ} \mathrm{C}$. $[\alpha]_{D}^{20}=+45.9^{\circ}$ (chloroform, $c=4$ ).

Analysis: Calculated for $\mathrm{C}_{17} \mathrm{H}_{24} \mathrm{O}_{12}$ : C, 48.57; $\mathrm{H}, 5.74 ; \mathrm{CH}_{3} \mathrm{CO}, 51.20$. Found: $\mathrm{C}, 48.4 ; \mathrm{H}, 5.6 ; \mathrm{CH}_{3} \mathrm{CO}, 51.4$.

To show the relation of the new pentaacetate to the previously known hexaacetate, $1 \mathrm{~g}$ of the pentaacetate was acetylated at room temperature with $6 \mathrm{ml}$ of acetic anhydride and $7 \mathrm{ml}$ of pyridine. The product crystallized when poured into ice water with stirring. After recrystallization from alcohol, the compound gave a specific rotation of $+86^{\circ}$ in comparison with a specific rotation of $+87^{\circ}$ for hexaacetyl$\alpha$-D-glucoheptulose. The pentaacetate therefore appears to be a derivative of the pyranose modification of the sugar.

\section{OCTAACETYLLACTULOSE ${ }^{11}$}

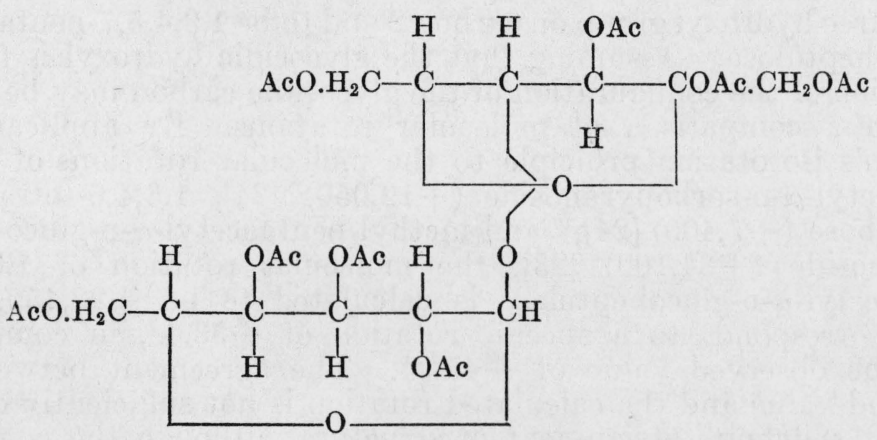

The ketose obtained from lactose by means of the Lobry de BruynVan Ekenstein interconversion reaction was first prepared by Montgomery and Hudson and called lactulose [25]. In a previous publication from this laboratory [26] data were presented that indicate that lactulose crystallizes from solution in the form of a furanose modifica- 
tion. The crystallization of a furanose from a pyranose-furanose equilibrium mixture is unique, and confirmation of the furanose structure is desirable. The determination of the structure of a crystalline sugar is complicated by the fact that isomeric changes take place on dissolution. During acetylation with pyridine and acetic anhydride at low temperatures, isomeric changes occur to only a limited degree, and the product usually contains the same modification as the parent sugar. Acetylation of finely powered lactulose with pyridine gave a crystalline octaacetate. As the parent sugar has been shown to be a furanose, it is probable that the acetate is a furanose derivative. Data are not available for calculating the optical rotation of the furanose acetate, but comparisons with certain pyranose derivatives appear to indicate that the compound is not a pyranose. The molecular rotation of octaacetyl- $\alpha$-lactulopyranose may be calculated from the molecular rotations of octaacetyl- $\alpha$-lactose $(+36,400)$ [27], pentaacetyl- $\alpha$-D-glucopyranose $(+39,700)[28]$, and of pentaacetyl- $\alpha$-D-fructopyranose $(+18,500)$ [29], to be $+15,200$. This value corresponds to a specific rotation of $+22.4^{\circ}$. From the moleeular rotations of the corresponding beta compounds (octaacetyl$\beta$ lactose $(-3,200)[27]$, pentaacetyl- $\beta$-D-glucopyranose $(+1,500)$ [28], and pentaacetyl- $\beta$-D-fructopyranose $(-47,200)[30])$, the specific rotation of octaacetyl- $\beta$-lactulopyranose is calculated to be $-76.5^{\circ}$. Hence the specific rotation of our acetate, $-6.6^{\circ}$, does not agree with either of the rotations calculated for the pyranose derivatives. Although it seems possible that the compound, like the parent sugar, is a furanose derivative, assignment of a definite structure is not attempted at this time.

Preparation of octaacetyllactulose.-Twenty grams of finely powdered crystalline lactulose was acetylated at approximately $-15^{\circ} \mathrm{C}$ with a mixture consisting of $92 \mathrm{ml}$ of acetic anhydride and $140 \mathrm{ml}$ of pyridine. After 2 days the reaction mixture was poured into ice water and the product was extracted with chloroform. The chloroform extract was concentrated in a vacuum still, and the sirupy residue was dissolved in acetone. The acetone solution was concentrated to a sirup, from which a crystalline product was obtained by the addition of ethyl alcohol. The new acetate formed mats of long hair-like crystals (fig. 2, D) which were difficult to separate by filtration. However, when the substance was allowed to crystallize slowly and in motion from hot ethyl alcohol, or from hot water, it formed short needles (fig. 2, $E$ ) which could be separated easily. The acetate, when dry, readily acquired an electrostatic /charge, land tended to scatter. After several recrystallizations from hot ethyl alcohol, it melted at $138^{\circ} \mathrm{C} .[\alpha]_{D}^{20}=-6.6^{\circ}($ chloroform, $c=4)$. These constants were unchanged by further recrystallization from lethyl alcohol, isopropyl alcohol, carbon tetrachloride, or water.

Analysis: Calculated for $\mathrm{C}_{28} \mathrm{H}_{38} \mathrm{O}_{19}: \mathrm{C}, 49.56 ; \mathrm{H}, 5.64 ; \mathrm{CH}_{3} \mathrm{CO}$, 50.75. Found: $\mathrm{C}, 49.5 ; \mathrm{H}, 5.5 ; \mathrm{CH}_{3} \mathrm{CO}, 50.9$.

The authors express their appreciation to Kenneth Fleischer for making the carbon, hydrogen, acetyl, and methoxyl determinations reported in this paper. 


\section{REFERENCES}

[1] H. S. Isbell, Ann. Rev. Biochem. 9, 65 (1940).

[2] H. L. Frush and H. S. Isbell, J. Research NBS 2\%, 413 (1941) RP1429.

[3] E. Pacsu, J. Am. Chem. Soc. 55, 2451 (1933); 57, 745 (1935); 61, 1654 (1939).

[4] E. Fischer, Liebigs Ann. Chem. 2\%0, 87 (1892).

[5] H. S. Isbell, J. Research NBS 18, 505 (1937) RP990.

[6] H. S. Isbell and H. L. Frush, J. Research NBS 24, 125 (1940) RP1274.

[7] H. S. Isbell, BS J. Research 5, 741 (1930) RP226.

[8] H. S. Isbell, J. Am. Chem. Soc. 56, 2789 (1934).

[9] W. Koenigs and E. Knorr, Ber. deut. chem. Ges. 34, 957 (1901).

[10a] C. S. Hudson, BS Sci. Pap. 21, 241 (1926) S533.

[10b] F. J. Bates and Associates, Polarimetry, saccharimetry, and the sugars, Circular National Bureau of Standards C440 (1942).

[11] C. S. Hudson and E. Yanovsky, J. Am. Chem. Soc. 38, 1575 (1916).

[12] W. N. Haworth, E. L. Hirst, and M. Stacey, J. Chem. Soc. 1931, 2864.

[13] H. S. Isbell, BS J. Research \%, 1115 (1931) RP392.

[14] V. Ettel, Coliection Czechoslov. Chem. Commun. 4, 504 (1932).

[15] H. S. Isbell, J. Research NBS 20, 97 (1938) RP1069.

[16] W. W. Pigman and H. S. Isbell, J. Research NBS 19, 189 (1937) RP1021.

[17] C. S. Hudson, J. Am. Chem. Soc. 48, 1434 (1926).

[18] C. S. Hudson and H. O. Parker, J. Am. Chem. Soc. 37, 1589 (1915).

[19] E. M. Montgomery and C. S. Hudson, J. Am. Chem. Soc. 56, 2463 (1934).

[20] F. B. LaForge, J. Biol. Chem. 41, 251 (1920).

[21] H. S. Isbell, J. Research NBS 19, 644 (1937) RP1052.

[22] H. S. Isbell, BS J. Research 8, 1 (1932) RP396.

[23] W. C. Austin, J. Am. Chem. Soc. 54, 1925 (1932).

[24] H. H. Schlubach and G. Graefe, Liebigs Ann. Chem. 532, 211 (1937).

[25] E. M. Montgomery and C. S. Hudson, J. Am. Chem. Soc. 52, 2101 (1930).

[26] H. S. Isbell and W. W. Pigman, J. Research NBS 20, 773 (1938) RP1104.

[27] C. S. Hudson and J. M. Johnson, J. Am. Chem. Soc. 3\%, 1270 (1915).

[28] C. S. Hudson and J. K. Dale, J. Am. Chem. Soc. 37, 1264 (1915).

[29] E. Pacsu and F. B. Cramer, J. Am. Chem. Soc. 5\%, 1945 (1935).

[30] C. S. Hudson and D. H. Brauns, J. Am. Chem. Soc. 3\%, 1283 (1915).

Washington, March 20, 1945. 